$\underset{\text { clinical }}{\text { nephron }}$

Practice
Nephron 2018;140:81-85

DOI: $10.1159 / 000489576$
Received: April 3, 2018

Accepted after revision: April 22, 2018

Published online: May 23, 2018

\title{
Improving Translation from Preclinical Studies to Clinical Trials in Acute Kidney Injury
}

\author{
Marco Fiorentino ${ }^{a}$ b John A. Kellum ${ }^{a}$ \\ ${ }^{a}$ Center for Critical Care Nephrology, Department of Critical Care Medicine, CRISMA (Clinical Research, Investigation, \\ and System Modeling of Acute Illness) Center, University of Pittsburgh, Pittsburgh, PA, USA; ${ }^{b}$ Department of \\ Emergency and Organ Transplantation, Nephrology, Dialysis and Transplantation Unit, University of Bari, Bari, Italy
}

\section{Keywords}

Acute kidney injury · Preclinical studies · Biomarkers

\section{Abstract}

Background: Several cellular and molecular targets and mechanisms have been investigated in preclinical studies of acute kidney injury (AKI), but translation in successful clinical studies has failed to date. This article reviews many issues that have limited this and the potential future perspectives in AKI prevention and treatment. Summary: Preclinical models of AKI should closely mimic the complexity of human AKI, considering the importance of several comorbidities in determining the clinical course and outcomes in the human disease. Moreover, studies should test novel interventions in models where AKI is already established, instead of focusing only at primary prevention. AKI definitions and endpoints in animal studies should be similar to those applied in clinical studies; in particular, AKI biomarkers should be implemented to guide patient selection for clinical trials and monitor intervention efficacy. In this scenario, cell-cycle arrest biomarkers have been widely investigated as AKI predictors in both preclinical and clinical studies and they serve as useful tools for future interventional studies. A better understanding of human AKI through a large collection of biological samples and kidney biopsies and omics applications, and an iterative relationship between preclinical and clinical studies are critical steps to improve future preclinical models and clinical trials. Finally, given the great variability in clinical manifestation of $\mathrm{AKI}$, a strong collaboration between research centers and industry is recommended. Key messages: Several methodological issues have hampered the translation of basic research findings in clinical studies, and overcoming these obstacles is necessary to achieve success.

(c) 2018 S. Karger AG, Basel

Contribution from the AKI and CRRT 2018 Symposium at the 23rd International Conference on Advances in Critical Care Nephrology, Manchester Grand Hyatt, San Diego, CA, USA, February 26 - March 1 , 2018. This symposium was supported in part by the NIDDK funded University of Alabama at Birmingham-University of California San Diego O'Brien Center for Acute Kidney Injury Research (P30 DK079337).

\section{KARGER}

(c) 2018 S. Karger AG, Basel

E-Mail karger@karger.com

www.karger.com/nef
John A. Kellum, MD, MCCM

Department of Critical Care Medicine, University of Pittsburgh

3347 Forbes Avenue, Suite 220

Pittsburgh, PA 15213 (USA)

E-Mail kellum@pitt.edu 


\section{Introduction}

Acute kidney injury (AKI) is a common clinical condition, associated with increased length of hospital stay, health care costs, morbidity and mortality in critically ill patients [1]. Several advances have been made in the early recognition of clinical AKI and its risk factors. Similarly, several cellular and molecular mechanisms and potential targets of AKI have been investigated in recent years in various preclinical models [2]. However, these promising findings were not successfully confirmed in clinical studies and no effective AKI therapies have been approved to date. Differences between preclinical and clinical studies represented one of the most important barriers to translation into clinical practice [3]. The purpose of this article is to discuss the lack of translation between animal and human AKI models and to propose future directions for improving translational AKI research.

\section{Disconnection between Preclinical and Clinical AKI Studies: Why?}

The purpose of using a preclinical model of AKI is to provide novel insights into the pathogenetic mechanisms and pathways implicated in the development of AKI. Moreover, the identification of new diagnostic biomarkers and specific therapeutic targets should improve the ability to predict and diagnose the disease and inform on the design of future clinical trials [4]. There are several reasons for the failure of this translational approach. First, a preclinical model must closely mimic the human condition. Human AKI is a systemic and nonspecific clinical syndrome that can result from different potential etiologies and a great variability in the clinical manifestation [5]. Sepsis is the most common cause of AKI; however, in septic patients, other factors like age, comorbidities (diabetes, cardiovascular disease, chronic kidney disease), and potential exposures (nephrotoxic drugs, contrast media, fluid administration) may influence AKI development and progression. These risk factors have been poorly considered in setting animal models for AKI. The Acute Dialysis Quality Initiative XIII Working Group proposed the development of context-specific preclinical models that not only replicate the exposures but also consider the human complexity [2]. Interesting findings from single experimental models should be validated in more realistic models and then tested in human AKI.
Second, the majority of AKI cases in the clinical practice are not preventable and most patients already have AKI at the time of presentation to medical attention [6]. By contrast, most preclinical studies mainly focused on a prophylactic approach, where the protective agents are administered before the renal insult (primary prevention) [7]. In this setting, a secondary prevention approach, where a potential treatment is given when AKI is already established, might be more realistic, and closer to the human condition [2].

Third, the selection of appropriate and similar AKI definitions and endpoints for outcome measures was an important limitation in translating preclinical findings in clinical studies in AKI. There is an important disconnect between preclinical and clinical interventional studies concerning the setting; most clinical studies evaluate AKI in patients undergoing cardiac surgery or in the context of contrast-associated AKI, while preclinical studies focused more on ischemia-reperfusion injury and drug-induced AKI [3]. Moreover, an important heterogeneity in the definitions of AKI between preclinical and clinical studies has been demonstrated and only few studies included AKI biomarkers in the outcomes. Most animal and human studies rely on changes in serum creatinine, need for dialysis, and mortality, but each of these outcomes measures is characterized by several issues. For example, the definition of the reference creatinine to assess the degree of creatinine changes and AKI stages is controversial [4]. Dialysis use is often influenced by clinical judgment, and several factors and comorbidities determine mortality risk. These potential confounders should be considered. Finally, AKI biomarkers may also have a role in "hardening" clinical endpoints, since tissue is not obtained in clinical trials.

Given all these limitations, the redesign of preclinical studies is urgently needed to avoid false positive discoveries and improve the clinical relevance and the translation of preclinical findings [8]. Similarly, clinical trials could improve by taking these limitations into account.

\section{AKI Biomarkers: A Tool for Translational Research in AKI}

The development and implementation of new therapeutic agents first require an accurate ability to predict and diagnose AKI. Since serum creatinine and urine output are late and functional markers, several novel AKI biomarkers have been identified in both preclinical
Fiorentino/Kellum 
Table 1. Future directions in translational research in AKI

Future directions

- Include human clinical factors (age, comorbidities) in the design of preclinical models of AKI (context-specific preclinical models).

- Secondary prevention approaches should be preferred to primary prevention.

- Systematic collection of biological samples and kidney tissue (databanks) for improving the knowledge of similarities and differences between animal and human AKI.

- Assess specific AKI endophenotypes through application of omic technologies in both preclinical and clinical studies.

- Integrate AKI biomarkers in the definitions and outcomes in both preclinical and clinical studies.

- Results, both positive or negative, from clinical studies should inform and improve the setting of future preclinical models.

- Networks and collaborations between centers to share information on AKI endophenotypes.

and clinical studies in the last few years. In particular, great efforts have been made to identify biomarkers of renal damage, or even stress, which may enable physicians to detect the disease early and limit its extent. These biomarkers may also play important roles in the design of new trials for investigational drugs by providing enrichment [9]. By excluding patients who are unlikely to develop AKI, we can apply our interventions to those at highest risk, thus reducing costs and potential toxicity. In clinical trials, we can dramatically reduce sample size and therefore improve both feasibility and safety.

While several novel biomarkers may have utility for enrichment, the cell cycle arrest biomarkers tissue inhibitor of metalloproteinases-2 (TIMP-2) and insulinlike growth factor binding protein 7 (IGFBP7) appear particularly promising, with evidence in both animal and human studies. Both TIMP-2 and IGFBP7 are inducers of G1 cell-cycle arrest that occurs early after cellular stress [10]. Temporary G1 cell-cycle arrest represents a protective mechanism to avoid the replication of injured cells and, consequently, reduce kidney damage. However, a prolonged cell-cycle arrest can result in a fibrotic phenotype switch that characterizes the maladaptive response leading to chronic kidney disease [11]. Together these markers outperform other existing AKI biomarkers in predicting the development of moderatesevere AKI in the next $12 \mathrm{~h}[12]$, and the product of ([TIMP-2]·[IGFBP7]) has been approved for clinical use. ([TIMP-2]·[IGFBP7]) rapidly increases in response to several different insults in patients who develop AKI [13] and also correlates with adverse long-term outcomes [14]. Moreover, their performance has been tested and validated also in animal studies. In a rat model of AKI secondary to cecal ligation and puncture, ([TIMP2]·[IGFBP7]) was significantly higher in animals that developed AKI and they also predicted mortality [15].

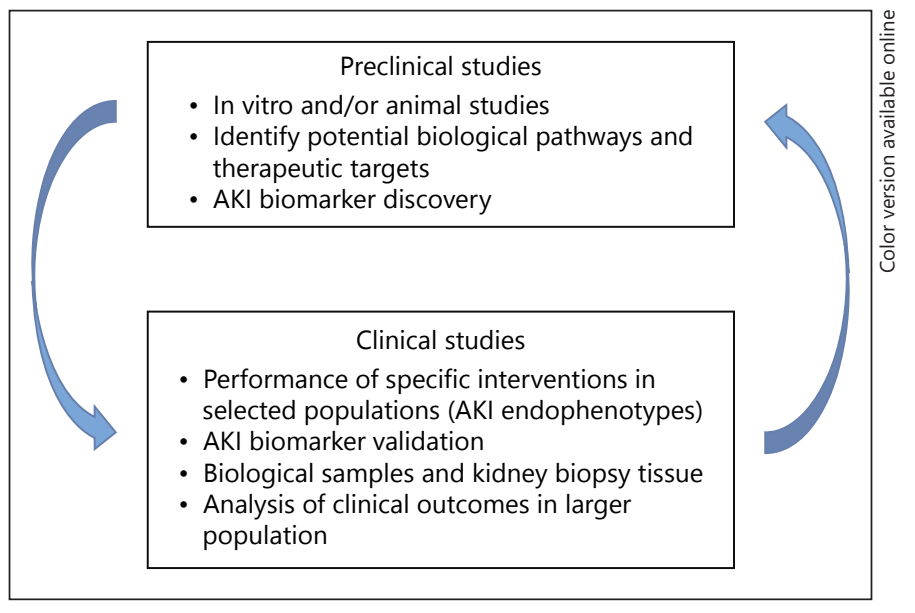

Fig. 1. The dynamic process from preclinical to clinical studies and back. Preclinical in vitro or in vivo studies are needed to identify new cellular and molecular mechanisms in the pathogenesis of AKI and potential biomarkers of renal stress/damage. Findings from basic research should inform on the design of clinical studies, where potential interventions should be tested in selected patients, characterized by specific AKI endophenotypes that are more likely to respond to treatment. AKI biomarkers should be validated and used in clinical studies to guide patient selection and monitor treatment effects. Both positive or negative results from clinical studies will inform on the design of future preclinical models. AKI, acute kidney injury.

\section{Future Directions}

Despite several discoveries in cellular and molecular targets implicated in the pathogenesis of AKI, a successful clinical translation of these findings has failed, and none have yet successfully translated into clinical practice. Several steps should now be taken to improve translational research in AKI (Table 1). A first step should be a better understanding of human AKI; in this scenario, future studies should systematically collect biological samples 
and kidney biopsy tissue in order to discover new pathogenetic pathways in the human disease [8]. In the era of precision medicine, the application of high-throughput technologies may help in assessing similarities and differences in the pathogenesis of AKI between animal and human models, identifying specific AKI endophenotypes and, consequently, in improving the design of clinical trials (e.g., selecting those patients with high probability of treatment success or excluding patients with a low probability of benefit). The increased knowledge on human AKI should also be critical in optimizing preclinical animal models that need to include clinical risk factors (comorbidities) influencing patient susceptibility to the disease [4]. Hence, the traditional "bench to bedside" approach should be replaced by a more dynamic process, where clinical studies should be designed on the basis of preclinical findings and their results should be used to develop more appropriate preclinical models that closely mimic the human disease (Fig. 1). In this scenario, since human AKI is hard to prevent in clinical practice, it is also important that future preclinical studies should prioritize secondary prevention, where candidate interventions are administered when AKI is already present, at least by biomarkers, instead of using them prophylactically [2]. In order to achieve these goals, given the high heterogeneity of AKI, there is a critical need to establish collaborations among centers and develop networks and databanks to collect clinical and biological information on AKI patients characterized by specific history of disease, clinical course, and, where possible, histopathology [4]. Importantly, a strong relationship between academia and industry is urgently recommended for improving the design of both preclinical and clinical studies [2]. The use of standardized definitions of AKI among preclinical and clini- cal studies and the inclusion of AKI biomarkers both for patient selection, and even possibly as endpoints, is crucial to predict and monitor the response to novel AKI therapies [3].

\section{Conclusions}

AKI is still a severe clinical condition in critically ill patients with high morbidity and mortality due to the absence of specific therapies for AKI. A translational approach in AKI research is required to develop effective interventions and improve AKI outcomes in the clinical setting. The improvement in the knowledge of human AKI, the development of more clinically relevant preclinical models, the introduction of novel approach (omics), the availability of novel biomarkers and networks, and collaborations between academia and industry are the next steps in facilitating translational research in AKI.

\section{Disclosure Statement}

This work was funded by institutional grants. The content is solely the responsibility of the authors. J.A.K. reports consulting and/or grant support from various companies developing AKI biomarkers, including Astute Medical, Alere and BioPorto. M.F. reports having received consulting fees from Bioporto.

\section{Funding Statement}

This study was funded in part by the Center for Critical Care Nephrology, University of Pittsburgh, Pittsburgh, PA, USA and the Nephrology, Dialysis and Transplantation Unit, University of Bari, Bari, Italy.

\section{References}

1 Kellum JA, Bellomo R, Ronco C: Kidney attack. JAMA 2012;307:2265-2266.

-2 Agarwal A, Dong Z, Harris R, Murray P, Parikh SM, Rosner MH, Kellum JA, Ronco C: Cellular and molecular mechanisms of AKI. J Am Soc Nephrol 2016;27:12881299.

-3 Fiorentino M, Castellano G, Kellum JA: Differences in acute kidney injury ascertainment for clinical and preclinical studies. Nephrol Dial Transplant 2017;32:17891805.

4 Zarjou A, Sanders PW, Mehta RL, Agarwal A: Enabling innovative translational research in acute kidney injury. Clin Transl Sci 2012;5: 93-101.
5 Kellum JA: Why are patients still getting and dying from acute kidney injury? Curr Opin Crit Care 2016;22:513-519.

6 Kellum JA, Chawla LS, Keener C, Singbartl K, Palevsky PM, Pike FL, Yealy DM, Huang DT, Angus DC: The effects of alternative resuscitation strategies on acute kidney injury in patients with septic shock. Am J Respir Crit Care Med 2016;193:281-287.

7 Palevsky PM, Molitoris BA, Okusa MD, Levin A, Waikar SS, Wald R, Chertow GM, Murray PT, Parikh CR, Shaw AD, Go AS, Faubel SG, Kellum JA, Chinchilli VM, Liu KD, Cheung AK, Weisbord SD, Chawla LS, Kaufman JS, Devarajan P, Toto RM, Hsu CY, Greene T, Mehta RL, Stokes JB, Thompson AM, Thomp- son BT, Westenfelder CS, Tumlin JA, Warnock DG, Shah SV, Xie Y, Duggan EG, Kimmel PL, Star RA: Design of clinical trials in acute kidney injury: report from an NIDDK workshop on trial methodology. Clin J Am Soc Nephrol 2012;7:844-850.

8 Zuk A, Palevsky PM, Fried L, Harrell FE Jr, Khan S, McKay DB, Devey L, Chawla L, de Caestecker M, Kaufman JS, Thompson BT, Agarwal A, Greene T, Okusa MD, Bonventre JV, Dember LM, Liu KD, Humphreys BD, Gossett D, Xie Y, Norton JM, Kimmel PL, Star RA: Overcoming translational barriers in acute kidney injury: a report from an NIDDK workshop. Clin J Am Soc Nephrol 2018;9:pii. 
9 Kellum JA, Devarajan P: What can we expect from biomarkers for acute kidney injury? Biomark Med 2014;8:1239-1245.

10 Kellum JA, Chawla LS: Cell-cycle arrest and acute kidney injury: the light and the dark sides. Nephrol Dial Transplant 2016;31:1622.

11 Yang L, Besschetnova TY, Brooks CR, Shah JV, Bonventre JV: Epithelial cell cycle arrest in G2/M mediates kidney fibrosis after injury. Nat Med 2010;16:535-543.

12 Kashani K, Al-Khafaji A, Ardiles T, Artigas A, Bagshaw SM, Bell M, Bihorac A, Birkhahn R, Cely CM, Chawla LS, Davison DL, Feldkamp
T, Forni LG, Gong MN, Gunnerson KJ, Haase M, Hackett J, Honore PM, Hoste EA, JoannesBoyau O, Joannidis M, Kim P, Koyner JL, Laskowitz DT, Lissauer ME, Marx G, McCullough PA, Mullaney S, Ostermann M, Rimmele T, Shapiro NI, Shaw AD, Shi J, Sprague AM, Vincent JL, Vinsonneau C, Wagner L, Walker MG, Wilkerson RG, Zacharowski K, Kellum JA: Discovery and validation of cell cycle arrest biomarkers in human acute kidney injury. Crit Care 2013;17:R25.

13 Ostermann M, McCullough PA, Forni LG, Bagshaw SM, Joannidis M, Shi J, Kashani K, Honore PM, Chawla LS, Kellum JA: Kinetics of urinary cell cycle arrest markers for acute kidney injury following exposure to potential renal insults. Crit Care Med 2018;46:375-383. 14 Koyner JL, Shaw AD, Chawla LS, Hoste EA, Bihorac A, Kashani K, Haase M, Shi J, Kellum JA: Tissue inhibitor metalloproteinase-2 (TIMP-2) IGF-binding protein-7 (IGFBP7) levels are associated with adverse long-term outcomes in patients with AKI. J Am Soc Nephrol 2015;26:1747-1754.

15 Peng ZY, Zhou F, Kellum JA: Cross-species validation of cell cycle arrest markers for acute kidney injury in the rat during sepsis. Intensive Care Med Exp 2016;4:12. 